\title{
Relating disease and predation: equilibria of an epidemic model
}

\author{
Manuel Delgado, Mónica Molina-Becerra and Antonio Suárez \\ Dpto Ecuaciones Diferenciales y Análisis Numérico, \\ Universidad de Sevilla, Apdo. de Correos 1160, \\ 41080 Sevilla, Spain \\ email: madelgado@us.es, monica@us.es, suarez@us.es \\ Dedicated to the memory of Prof. Ovide Arino
}

Short title: Relating disease and predation

Corresponding author:

Mónica Molina-Becerra

Dpto Ecuaciones Diferenciales y Análisis Numérico, Universidad de Sevilla, Apdo. de Correos 1160, 41080 Sevilla, Spain

e-mail: monica@us.es

Fax: (34) 954552898

Tel: (34) 954557981

Contract/grant sponsor: M.E.C. (Spain, Feder); Contract/grant number: BFM2003-06446 


\begin{abstract}
In this paper, we would like compare the spread of an infectious disease in a population without the influence of a predator and under its influence. We show that it is possible to control an epidemic in a population with the help of predators.
\end{abstract}


Key words: Predator, Prey, Epidemic, Stability, Dulac's Criterion.

\section{Introduction}

After the pioneering works of Lotka [1] and Von Foerster [2] population dynamic models depending on the age have been analyzed extensively in the last years. Later, Gurtin and MacCamy [3] extended the model introducing the total population, (see the monograph of Webb [4] for a survey of nonlinear models of populations with age structure).

On the other hand, models of interacting biological species have been extensively studied, epidemic models (see [5] for a survey), predator-prey models with and without disease in the species, (see [6-9] to mention a few)... Recently, Chattopadhyay et al. [10] studied a predatorprey model with disease in the prey. In this work the rate of birth, death,... are assumed constants.

In [11], we studied a nonlinear age-dependent epidemic model in which a predator interacts. We assumed that the prey is affected by an infectious disease which weakens the prey and its susceptibility to predation increases. The predator grows according to a logistic law. We considered a general framework of hypotheses (different rate of mortality for the infective and susceptible individuals, a general force of infection, vertical transmission,...) and we studied the asymptotic behavior of disease-free equilibria. We considered the following system:

$$
\left\{\begin{array}{l}
\frac{\partial i}{\partial t}+\frac{\partial i}{\partial a}+\mu_{1}(a, P(t)) i(a, t)=K(a) I(t) s(a, t)-M_{1} i(a, t) Y(t), \\
\frac{\partial s}{\partial t}+\frac{\partial s}{\partial a}+\mu_{2}(a, P(t)) s(a, t)=-K(a) I(t) s(a, t)-M_{2} s(a, t) Y(t), \\
\frac{d Y}{d t}=m Y(t)-n Y^{2}(t)+\varepsilon M_{1} I(t) Y(t)+\varepsilon M_{2} S(t) Y(t), \\
i(a, 0)=i_{0}(a), s(a, 0)=s_{0}(a), Y(0)=Y_{0}, \\
i(0, t)=q \int_{0}^{\infty} \beta(a, P(t)) i(a, t) d a, \\
s(0, t)=\int_{0}^{\infty} \beta(a, P(t))(s(a, t)+(1-q) i(a, t)) d a,
\end{array}\right.
$$

where $i(a, t)$ and $s(a, t)$ denote the age-density for infective and susceptible preys of age $a$ at time $t$, respectively, and $Y(t)$ denotes the density of predator. Moreover, $I(t), S(t)$ and $P(t)$ denote the total population of infectives, susceptibles and prey, respectively, namely

$$
I(t)=\int_{0}^{\infty} i(a, t) d a, S(t)=\int_{0}^{\infty} s(a, t) d a \text { and } P(t)=I(t)+S(t) .
$$


The functions $\mu_{1}$ and $\mu_{2}$ represent the death rate of the infective and susceptible individuals, respectively, and $\beta$, the birth rate. $K$ is the force of infection. And the positive constants $m, q \in[0,1], \varepsilon, M_{1}>0$ and $M_{2}>0$ denote the intrinsic birth rate of the predator, the vertical transmission, the coefficient of conversion of the prey into predator, the predation rate on infected and on susceptible prey, respectively. And $D:=m / n$, where $D$ is the carrying capacity of the environment, which is usually determined by the available sustaining resources. For a deeper discussion of the model we refer the reader [11].

The most interesting result obtained in [11] is the possibility to have a stable disease-free equilibrium with a positive susceptible prey, i.e. the predator would help to remove the disease. The difficulties arise from finding another equilibrium point because of the age dependence.

In this paper we consider the model studied in [11] but assuming that all the coefficients are not depending on age. Thus integrating with respect to age the model (1) (supposing all the coefficients are not depending on age) we obtain the following system:

$$
\left\{\begin{array}{l}
I^{\prime}=q \beta(P) I-\mu_{1}(P) I+K I S-M_{1} I Y, \\
S^{\prime}=\beta(P)(S+(1-q) I)-\mu_{2}(P) S-K I S-M_{2} S Y, \\
Y^{\prime}=m Y-n Y^{2}+\varepsilon M_{1} I Y+\varepsilon M_{2} S Y .
\end{array}\right.
$$

Hence, our purpose is to study the global dynamical behavior of the model (2).

We suppose the following mathematical hypotheses: $\mu_{i}:[0, \infty) \rightarrow[0, \infty)$ for $i=1,2$ such that $\mu_{i}$ is continuously differentiable, monotonically increasing and $\mu_{i}(P) \rightarrow \infty$ as $P \rightarrow \infty$. We are assuming that the disease affects the death rate, so we have that $\mu_{1} \geq \mu_{2} . \beta:[0, \infty) \rightarrow[0, \infty)$ is continuously differentiable, monotonically decreasing and $\beta(P) \rightarrow 0$ as $P \rightarrow \infty$. These rates correspond to a mortality and natality process in a harsh environment. Since we consider the case when the predator mainly eats the infected prey, we assume that $M_{1}>M_{2}$.

An outline and summary are as follow: Section 2 is devoted to the study of (2) without predator. And we convert our three-dimensional system to a two-dimensional one. This allows us to use results only applicable to two-dimensional system, for instance the PoincaréBendixson Theorem.

We show that the trivial solution $(0,0)$ is globally asymptotically stable (GAS) if $\mu_{2}(0)>\beta(0)$. On the other hand, if $\mu_{2}(0)<\beta(0)$ there exist $0<\mathcal{K}_{1}, \mathcal{K}_{2}, \mathcal{K}_{3}<+\infty$ (see Th. 2.2 and 2.7 and Corollary 2.3) such that

- if $K<\mathcal{K}_{1}$ then there exists a unique equilibrium $\left(0, P_{S}\right)$ and it is GAS.

- if $K>\mathcal{K}_{2}$ then there exist an odd number of equilibria $\left(I^{*}, S^{*}\right)$ and they are locally 
asymptotically stable (LAS).

- if $K>\mathcal{K}_{3}$ then there exists a unique equilibrium $\left(I^{*}, S^{*}\right)$ and it is GAS.

The complete model (2) is analyzed in Section 3. We prove, under some conditions, the existence of equilibria $\left(I^{*}, S^{*}, Y^{*}\right)$ and their local stability. On the other hand, if $M_{2} m / n>$ $\beta(0)-\mu_{2}(0)$ then the equilibrium $(0,0, m / m)$ is GAS; while if $M_{2} m / n<\beta(0)-\mu_{2}(0)$ there exists $\mathcal{M}(K)>0$ such that if $M_{1}>\mathcal{M}(K)$ then the equilibrium $\left(0, S^{*}, Y^{*}\right)$ is GAS (see Th. $3.3)$.

In Section 4, we summarize and give a biological interpretation to these results and by means of an example show that there exists a range of values of $K$ and $M_{1}$, such that, without the predator the disease persists and with the predator disappears.

\section{The model without predator}

In this section we consider the model (2) without predator, i.e.

$$
\left\{\begin{array}{l}
I^{\prime}=q \beta(P) I-\mu_{1}(P) I+K I S \\
S^{\prime}=\beta(P)(S+(1-q) I)-\mu_{2}(P) S-K I S \\
I(0)=I_{0}, S(0)=S_{0} .
\end{array}\right.
$$

From now on we write

$$
R_{0}:=\mu_{2}(0)-\beta(0), R_{1}:=\mu_{1}(0)-\beta(0)
$$

If $R_{0}<0$, then there exists $P_{S}>0$ such that

$$
\mu_{2}\left(P_{S}\right)=\beta\left(P_{S}\right)
$$

And if $R_{1} \leq 0$, then there exists $P_{1} \geq 0$ such that

$$
\mu_{1}\left(P_{1}\right)=\beta\left(P_{1}\right) \text { with } P_{1}<P_{S}
$$

The next result provides us the existence, uniqueness and boundedness of the positive solution of (3).

Proposition 2.1. There exists a unique positive solution of (3), which is global and uniformly bounded for each $\left(I_{0}, S_{0}\right) \in \mathbb{R}_{+}^{2}$. 
Proof. The Picard Theorem assures the existence and uniqueness of local solution for every $\left(I_{0}, S_{0}\right) \in \mathbb{R}_{+}^{2}$.

From (3), and using $P=I+S$, it is easy to check that

$$
\left(\beta(P(t))-\mu_{1}(P(t))\right) P(t) \leq P^{\prime}(t) \leq\left(\beta(P(t))-\mu_{2}(P(t))\right) P(t) .
$$

For $i=1,2$, we consider the systems

$$
(O D E)_{i} \quad\left\{\begin{array}{l}
\dot{x_{i}}=\left(\beta\left(x_{i}\right)-\mu_{i}\left(x_{i}\right)\right) x_{i} \\
x_{i}(0)=P_{0}:=I_{0}+S_{0}
\end{array}\right.
$$

By the comparison Theorem, we get that $x_{1}(t) \leq P(t) \leq x_{2}(t)$ on its respective definition intervals. Since $\beta$ is decreasing and $\mu_{2}$ is increasing,

1. If $R_{0} \geq 0$, then we have $\beta(r)<\mu_{2}(r) \forall r \in(0,+\infty)$ and $x_{2}(t) \leq P_{0}, \forall t \in[0,+\infty)$. So, $0<x_{1}(t) \leq P(t) \leq x_{2}(t) \leq P_{0} \forall t \in[0,+\infty)$.

2. If $R_{0}<0$, then $x_{2}(t) \leq \max \left\{P_{0}, P_{S}\right\} \forall t \in[0,+\infty)$. Hence $0<x_{1}(t) \leq P(t) \leq x_{2}(t) \leq$ $\max \left\{P_{0}, P_{S}\right\} \forall t \in[0,+\infty)$.

\subsection{Equilibria and stability}

Here we deal with the existence and stability of equilibria of $(3),\left(I^{*}, S^{*}\right)$. To do this we analyze the stationary system of (3),

$$
\left\{\begin{array}{l}
-q \beta\left(P^{*}\right) I^{*}+\mu_{1}\left(P^{*}\right) I^{*}=K I^{*} S^{*}, \\
-\beta\left(P^{*}\right)\left(S^{*}+(1-q) I^{*}\right)+\mu_{2}\left(P^{*}\right) S^{*}=-K I^{*} S^{*} .
\end{array}\right.
$$

Substituting $I^{*}=P^{*}-S^{*}$ and combining $(8)_{1}$ with $(8)_{2}$, we can rewrite (8) as

$$
\left\{\begin{array}{l}
\left(-q \beta\left(P^{*}\right)+\mu_{1}\left(P^{*}\right)-K S^{*}\right)\left(P^{*}-S^{*}\right)=0, \\
\left(\mu_{2}\left(P^{*}\right)-\mu_{1}\left(P^{*}\right)\right) S^{*}=\left(\beta\left(P^{*}\right)-\mu_{1}\left(P^{*}\right)\right) P^{*} .
\end{array}\right.
$$

Throughout the section, we introduce the following notations:

$$
\begin{aligned}
& S(P):=\frac{-q \beta(P)+\mu_{1}(P)}{K} \\
& T_{1}:=\max _{Q \in I_{1}}\left\{\mu_{1}^{\prime}(Q)-q \beta^{\prime}(Q)\right\} \text { where } I_{1}:=\left\{\begin{array}{l}
\left(P_{1}, P_{S}\right) \text { if } R_{1} \leq 0 \\
\left(0, P_{S}\right) \text { if } R_{1}>0 .
\end{array}\right.
\end{aligned}
$$


Theorem 2.2. There exists always the trivial equilibrium $(0,0)$. If $R_{0} \geq 0$ this point is the unique equilibrium. For $R_{0}<0$ there exists a disease-free equilibrium $\left(0, P_{S}\right)$ and

1. For $R_{1} \leq 0$, if

$$
K>K_{1}:=\max \left\{T_{1}, \frac{(1-q) \beta\left(P_{1}\right)}{P_{1}}\right\}
$$

there exists at least an endemic equilibrium $\left(I^{*}, S^{*}\right)$.

2. For $R_{1}>0$ and $K>T_{1}$, there exists at least an endemic equilibrium $\left(I^{*}, S^{*}\right)$ if, and only if,

$$
K>K_{2}:=\frac{\mu_{1}\left(P_{S}\right)-q \beta\left(P_{S}\right)}{P_{S}} .
$$

When the system (3) has endemic equilibria, then there is an odd number of endemic equilibria.

Proof. The proof of existence of the equilibrium $(0,0)$ and the disease-free equilibrium $\left(0, P_{S}\right)$ are straightforward. We will see that if $R_{0} \geq 0$ then there exists only the trivial equilibrium. In this case we have $\beta(P) \leq \mu_{2}(P)$ for all $P \geq 0$. Hence $(9)_{2}$ does not have solution unless $P^{*}=0$, since $S^{*}$ must be less than $P^{*}$.

Now, we will see the existence of the endemic equilibrium. We denote

$$
\Sigma:=\{P \in(0,+\infty) \text { such that } 0<S(P)<P\}
$$

By (9), we have $S^{*}=S\left(P^{*}\right)$, where $P^{*}>0$ verifies $J\left(P^{*}\right)=G\left(P^{*}\right)$, with $J$ and $G$ defined by

$$
J(P):=\left(\mu_{1}(P)-\beta(P)\right) P, \quad G(P):=\left(\mu_{1}(P)-\mu_{2}(P)\right) S(P) .
$$

Moreover, necessarily $P^{*}$ has to belong to $\Sigma$ to obtain $I^{*}=P^{*}-S^{*}>0$, thus, by $(9)_{2}, \beta\left(P^{*}\right)$ has to be greater than $\mu_{2}\left(P^{*}\right)$, hence $P^{*}<P_{S}$.

First we suppose $R_{1} \leq 0$. Since $\mu_{2} \leq \mu_{1}$, by $(9)_{2}$, we get that $\beta\left(P^{*}\right)<\mu_{1}\left(P^{*}\right)$, then $P^{*}>P_{1}$. Hence

$$
P^{*} \in\left(P_{1}, P_{S}\right)
$$

Since $K>K_{1}$ we have that $\left(P_{1}, P_{S}\right) \subseteq \Sigma$. Indeed, by (11) the function

$$
P \mapsto H(P):=S(P)-P
$$

is decreasing in $\left(P_{1}, P_{S}\right)$ and $S\left(P_{1}\right)-P_{1}<0$; and thus

$$
S(P)<P \text { for } P \in\left[P_{1}, P_{S}\right]
$$


It is easy to check that $J\left(P_{1}\right)=0<G\left(P_{1}\right)$ and, by $(15), J\left(P_{S}\right)>G\left(P_{S}\right)$, hence by Bolzano's Theorem we get an odd number of intersections of the two graphs.

Next, we assume $R_{1}>0$. Since $K>T_{1}$, then $H$ is decreasing. Supposing that $K \leq K_{2}$ then $H\left(P_{S}\right) \geq 0$, hence $H(P)>H\left(P_{S}\right) \geq 0$ for all $P \in\left(0, P_{S}\right)$. Then there does not exist any $P \leq P_{S}$ such that $P \in \Sigma$. Thus, there is not any endemic equilibrium.

Now, we assume $K>K_{2}$. In this case, we have $H(0)>0$ and $H\left(P_{S}\right)<P_{S}$, then there exists $P_{2} \in\left(0, P_{S}\right)$ such that $S\left(P_{2}\right)=P_{2}$, and

$$
S(P)>P \quad \forall P \in\left(0, P_{2}\right) \text { and } S(P)<P \quad \forall P \in\left(P_{2}, P_{S}\right] .
$$

Thus $J\left(P_{2}\right)<G\left(P_{2}\right)$ since $P_{2}<P_{S}$. And proceeding analogously to the former case, we see that there exist an odd number of intersections of the functions $J$ and $G$ in $\left(P_{2}, P_{S}\right)$.

Corollary 2.3. Suppose there exists at least an endemic equilibrium. Then if

$$
K>\max _{Q \in I_{1}}\left\{\frac{\left(\left(\mu_{1}(Q)-\mu_{2}(Q)\right)\left(-q \beta(Q)+\mu_{1}(Q)\right)\right)^{\prime}}{\left(\mu_{1}^{\prime}(Q)-\beta^{\prime}(Q)\right) Q+\left(\mu_{1}(Q)-\beta(Q)\right)}\right\},
$$

where $I_{1}$ is defined in (10), there exists a unique endemic equilibrium $\left(I^{*}, S^{*}\right)$.

Proof. It is not hard to check that $J^{\prime}(P)-G^{\prime}(P)>0$. This clearly gives us uniqueness of endemic equilibria.

Remark 2.4. Other conditions can be imposed to obtain uniqueness of endemic equilibrium (for instance on $\beta$ ).

Next we study the asymptotic behavior of the equilibria. To analyze the local stability we proceed by standard linearization techniques. Linearizing (3) around an equilibrium, $\left(I^{*}, S^{*}\right)$, we obtain a linear system whose eigenvalues $\lambda$ verify the characteristic equation

$$
\chi(\lambda) \equiv \lambda^{2}+\left(a_{1}+b_{2}\right) \lambda+\left(a_{1} b_{2}-a_{2} b_{1}\right)=0,
$$

where

$$
\begin{aligned}
& a_{1}:=-q \beta\left(P^{*}\right)+\mu_{1}\left(P^{*}\right)-K S^{*}+\left(-q \beta^{\prime}\left(P^{*}\right)+\mu_{1}^{\prime}\left(P^{*}\right)\right) I^{*} \\
& a_{2}:=\left(-q \beta^{\prime}\left(P^{*}\right)+\mu_{1}^{\prime}\left(P^{*}\right)-K\right) I^{*} \\
& b_{1}:=-\beta^{\prime}\left(P^{*}\right)\left(S^{*}+(1-q) I^{*}\right)-(1-q) \beta\left(P^{*}\right)+\mu_{2}^{\prime}\left(P^{*}\right) S^{*}+K S^{*} \\
& b_{2}:=-\beta\left(P^{*}\right)-\beta^{\prime}\left(P^{*}\right)\left(S^{*}+(1-q) I^{*}\right)+\mu_{2}\left(P^{*}\right)+\mu_{2}^{\prime}\left(P^{*}\right) S^{*}+K I^{*} .
\end{aligned}
$$


The two following results [12] are used from now on in this paper. Consider the following systems, see:

$$
\begin{aligned}
& \dot{x}=f(t, x), \\
& \dot{y}=g(y),
\end{aligned}
$$

where $f$ and $g$ are continuous and locally Lipschitz in $x \in \mathbb{R}^{n}$ and solutions exist for all positive time. Equation (18) is called asymptotically autonomous with limit equation (19) if $f(t, x) \rightarrow g(x)$ as $t \rightarrow \infty$ uniformly for $x \in \mathbb{R}^{n}$.

Lemma 2.5. Let e be a locally asymptotically stable equilibrium of (19) and $\omega$ be the $\omega$-limit set of a forward bounded solution $x(t)$ of (18). If $\omega$ contains a point $y_{0}$ such that the solution of (19), with $y(0)=y_{0}$ converges to e as $t \rightarrow \infty$, then $\omega=\{e\}$, i.e., $x(t) \rightarrow e$, as $t \rightarrow \infty$.

Corollary 2.6. If the solutions of system (18) are bounded and the equilibrium e of the limit system (19) is globally asymptotically stable, then any solution $x(t)$ of system (18) satisfies $x(t) \rightarrow e$, as $t \rightarrow \infty$.

Theorem 2.7. $\quad$ 1. The trivial equilibrium $(0,0)$ is globally asymptotically stable $(G A S)$ when $R_{0}>0$ and unstable when $R_{0}<0$.

2. The disease-free equilibrium $\left(0, P_{S}\right)$ is locally asymptotically stable $(L A S)$ when $K<K_{2}$ and unstable when $K>K_{2}$. Moreover, if

$$
K<K_{3}:=\left\{\begin{array}{l}
\frac{(1-q) \beta\left(P_{1}\right)}{P_{S}} \text { if } R_{1} \leq 0 \\
\frac{\mu_{1}(0)-q \beta(0)}{P_{S}} \text { if } R_{1}>0 .
\end{array}\right.
$$

then $\left(0, P_{S}\right)$ is $G A S$.

3. Under the assumption $K>T_{1}$ (defined in (10)) any endemic equilibrium $\left(I^{*}, S^{*}\right)$ is LAS. In fact, if $K>K_{2}$, it is GAS in the case of the uniqueness of the endemic equilibrium.

Proof. We first show the local stability.

It is easy to see that the roots of (17) for the equilibrium $(0,0)$ are $\lambda_{1}=q \beta(0)-\mu_{1}(0)$ and $\lambda_{2}=\beta(0)-\mu_{2}(0)$. Hence $R_{0}>0$ is the necessary and sufficient condition for local stability. Now, solving (17) for the equilibrium $\left(0, P_{S}\right)$ we see that the roots are $\lambda_{1}=K P_{S}-\mu_{1}\left(P_{S}\right)-$ $q \mu_{2}\left(P_{S}\right)$ and $\lambda_{2}=\left(\beta^{\prime}\left(P_{S}\right)-\mu_{2}^{\prime}\left(P_{S}\right)\right) P_{S}<0$. Hence the necessary and sufficient condition for local stability is $K<K_{2}$. 
Now we study the endemic equilibria $\left(I^{*}, S^{*}\right)$. Since $\left(I^{*}, S^{*}\right)$ verifies $(8)$, it is clear that $a_{1}, b_{1}, b_{2}>0$ and by $K>T_{1}$, we have $a_{2}<0$. Hence $\left(a_{1}+b_{2}\right)>0$ and $\left(a_{1} b_{2}-a_{2} b_{1}\right)>0$, thus by the Routh-Hurwitz condition we have that the endemic equilibria are always LAS.

Next we study the global stability.

For the equilibrium $(0,0)$, if $R_{0}>0$ then $\beta(P(t))<\mu_{2}(P(t)) \forall t \in \mathbb{R}_{+}$. Hence, from (7) $P(t) \rightarrow 0$ as $t \rightarrow \infty$. We observe that

$$
0 \leq S(t) \leq P(t)
$$

and so $S(t) \rightarrow 0$ as $t \rightarrow \infty$ and thus $I(t) \rightarrow 0$. Consequently if $R_{0}>0$, the equilibrium $(0,0)$ is GAS.

Now, supposing that $R_{1} \leq 0$. Since, we assume that $K<K_{3}$, and since $\beta$ and $\mu_{1}$ are continuous, then there exists $\delta>0$ such that

$$
q \beta\left(P_{1}-\delta\right)-\mu_{1}\left(P_{1}-\delta\right)+K\left(P_{S}+\delta\right)<0
$$

On the other hand, by (7) there exists $t_{0}>0$ such that $P_{1}-\delta \leq P(t) \leq P_{S}+\delta$, for $t \geq t_{0}$. Hence

$$
I^{\prime}(t) \leq\left(q \beta\left(P_{1}-\delta\right)-\mu_{1}\left(P_{1}-\delta\right)+K\left(P_{S}+\delta\right)\right) I(t)
$$

thus, $I(t) \rightarrow 0$ as $t \rightarrow \infty$.

In the same manner we can see this in the case $R_{1}>0$.

On the other hand

$$
S^{\prime}=\beta(S+I(t))(S+(1-q) I(t))-\mu_{2}(S+I(t)) S-K I(t) S .
$$

Hence this differential equation is asymptotically stable to $S^{\prime}=\left(\beta(S)-\mu_{2}(S)\right) S$. Using now $R_{0}<0$ and Corollary 2.6, $S(t) \rightarrow P_{S}$ as $t \rightarrow \infty$.

Now we assume the uniqueness of the endemic equilibrium $\left(I^{*}, S^{*}\right)$. We may apply the Dulac's criterion (see for instance [13]) to the two dimensional (3). Using the Dulac multiplier $1 /$ IS we obtain

$$
\begin{gathered}
\frac{\partial}{\partial I}\left(\frac{q \beta(I+S) I-\mu_{1}(I+S) I}{I S}+K\right)+\frac{\partial}{\partial S}\left(\frac{\beta(I+S)(S+(1-q) I)-\mu_{2}(I+S) S}{I S}-K\right) \\
=\beta^{\prime}(I+S)\left(\frac{1}{I}+\frac{1}{S}\right)-\frac{\mu_{1}^{\prime}(I+S)}{S}-\frac{\mu_{2}^{\prime}(I+S)}{I}-\frac{\beta(I+S)}{S^{2}}(1-q)<0 .
\end{gathered}
$$

Thus there is no periodic solution or cycle by Dulac's Criterion. Since we have that $(0,0)$ and $\left(0, P_{S}\right)$ are unstable and the endemic equilibrium is unique, it is GAS by the PoincaréBendixson Theorem [13]. 


\section{The model with predator}

In this section we consider the complete model (2).

Similarly to Proposition 2.1, we obtain global existence and uniqueness of the solution $(I(t), S(t), Y(t))$ of the system (2). Moreover, this solution is uniformly bounded.

\subsection{Equilibria and stability}

We begin by showing the existence of equilibrium, $\left(I^{*}, S^{*}, Y^{*}\right)$, of $(2)$. We consider the stationary system of (2), and proceeding analogously as the subsection 2.1 (substituting $I^{*}=P^{*}-S^{*}$ ), we obtain

$$
\left\{\begin{array}{l}
\left(-q \beta\left(P^{*}\right)+\mu_{1}\left(P^{*}\right)-K S^{*}+M_{1} Y^{*}\right)\left(P^{*}-S^{*}\right)=0 \\
\left(\mu_{2}\left(P^{*}\right)-\mu_{1}\left(P^{*}\right)+\left(M_{2}-M_{1}\right) Y^{*}\right) S^{*}=\left(\beta\left(P^{*}\right)-\mu_{1}\left(P^{*}\right)-M_{1} Y^{*}\right) P^{*} \\
m Y^{*}-n\left(Y^{*}\right)^{2}+\varepsilon M_{1} P^{*} Y^{*}+\varepsilon\left(M_{2}-M_{1}\right) S^{*} Y^{*}=0 .
\end{array}\right.
$$

Solving $(21)_{3}$ we find two possible solutions for $Y^{*}$

$$
Y^{*}=0, \quad Y^{*}=\left(m+\varepsilon M_{1} P^{*}+\varepsilon\left(M_{2}-M_{1}\right) S^{*}\right) / n
$$

The existence for the case $Y^{*}=0$ was studied in the above section. Hence we consider the equilibria for $Y^{*}=\left(m+\varepsilon M_{1} P^{*}+\varepsilon\left(M_{2}-M_{1}\right) S^{*}\right) / n$. From now on we write, for each $P \geq 0$

$$
\begin{aligned}
F_{i}(P) & :=-\beta(P)+\mu_{i}(P)+\frac{M_{i}}{n}\left(m+\varepsilon M_{i} P\right) \text { for } i=1,2, \\
S(P) & :=\frac{-q \beta(P)+\mu_{1}(P)+\frac{M_{1} m}{n}+\frac{\varepsilon M_{1}^{2}}{n} P}{K+\frac{M_{1} \varepsilon\left(M_{1}-M_{2}\right)}{n}}, \\
Y(P) & :=\frac{m+\varepsilon M_{1} P+\varepsilon\left(M_{2}-M_{1}\right) S(P)}{n}, \\
G_{i}(P) & :=\mu_{i}(P)+M_{i} Y(P)-\beta(P), \text { for } i=1,2, \\
J(P) & :=G_{1}(P) P, L(P):=\left(\mu_{1}(P)-\mu_{2}(P)+\left(M_{1}-M_{2}\right) Y(P)\right) S(P) .
\end{aligned}
$$

If $F_{2}(0)<0$, then there exists $P_{S Y}>0$ such that

$$
F_{2}\left(P_{S Y}\right)=0 .
$$

And if $F_{1}(0) \leq 0$, then there exists $Q_{1} \geq 0$ such that

$$
F_{1}\left(Q_{1}\right)=0 \text { with } Q_{1}<P_{S Y} .
$$


Let us introduce the following notation

$$
T_{2}:=\max _{Q \in I_{2}}\left\{-q \beta^{\prime}(Q)+\mu_{1}^{\prime}(Q)+\frac{\varepsilon M_{1} M_{2}}{n}\right\} \text { where } I_{2}:=\left\{\begin{array}{l}
\left(Q_{1}, P_{S Y}\right) \text { if } F_{1}(0) \leq 0 \\
\left(0, P_{S Y}\right) \text { if } F_{1}(0)>0 .
\end{array}\right.
$$

Theorem 3.1. There exists always the equilibrium $(0,0, m / n)$. If $F_{2}(0) \geq 0$ this one is the unique equilibrium.

For $F_{2}(0)<0$ there exists another disease-free equilibrium $\left(0, P_{S Y},\left(m+\varepsilon M_{2} P_{S Y}\right) / n\right)$ and,

1. For $F_{1}(0) \leq 0$, if

$$
K>\max \left\{T_{2}, \frac{(1-q) \beta\left(Q_{1}\right)}{Q_{1}}+\frac{\varepsilon M_{1}}{n}\left(M_{1}-M_{2}\right)\right\}
$$

there exists at least an endemic equilibrium $\left(I^{*}, S^{*}, Y^{*}\right)$.

2. For $F_{1}(0)>0$ and $K>T_{2}$, there exists at least an endemic equilibrium $\left(I^{*}, S^{*}, Y^{*}\right)$ if, and only if,

$$
K>K_{4}:=\frac{\mu_{1}\left(P_{S Y}\right)-q \beta\left(P_{S Y}\right)+\frac{M_{1}}{n}\left(m+\varepsilon M_{2} P_{S Y}\right)}{P_{S Y}} .
$$

Proof. As the proof is rather similar, although more involved, to the one of Theorem 2.2, we only sketch it.

The existence of the equilibrium $(0,0, m / n)$ and the disease-free equilibrium $\left(0, P_{S Y},(m+\right.$ $\left.\varepsilon M_{2} P_{S Y}\right) / n$ ) are straightforward from (21).

We are looking for an endemic equilibrium $\left(I^{*}, S^{*}, Y^{*}\right)$ such that $S^{*}=S\left(P^{*}\right), Y^{*}=Y\left(P^{*}\right)$ and $I^{*}=P^{*}-S\left(P^{*}\right)$, where $P^{*}>0$ has to verify

$$
J\left(P^{*}\right)=L\left(P^{*}\right)
$$

and $P^{*}$ belonging to the set $\Sigma:=\{P \in(0,+\infty)$ such that $0<S(P)<P\}$.

Since $L(P)$ is a positive function, then $G_{1}\left(P^{*}\right)>0$, and for $P \in \Sigma$

$$
L(P)=\left(G_{1}(P)-G_{2}(P)\right) S(P)<\left(G_{1}(P)-G_{2}(P)\right) P=J(P)-G_{2}(P) P,
$$

hence $G_{2}\left(P^{*}\right) \leq 0$. But, for $P \in \Sigma, Y(P)>\left(m+\varepsilon M_{2} P\right) / n$, hence $G_{2}(P)>F_{2}(P)$. Since $F_{2}$ is an increasing function, then a necessary condition for the existence of this equilibrium is $F_{2}(0)<0$, moreover $P^{*}$ has to be less than $P_{S Y}$.

In the case, $F_{1}(0) \leq 0$, then $Q_{1}<P^{*}<P_{S Y}$, and $\left(Q_{1}, P_{S Y}\right) \subseteq \Sigma$. Moreover $J\left(Q_{1}\right)<L\left(Q_{1}\right)$ and $J\left(P_{S Y}\right)>L\left(P_{S Y}\right)$. Bolzano's Theorem concludes the proof in this case. 
In the case $F_{1}(0)>0$, if $K \leq K_{4}$, there does not exist any $P \leq P_{S Y}$ such that $P \in \Sigma$. If $K>K_{4}$ then there exists $Q_{2} \in\left(0, P_{S Y}\right)$ such that

$$
S\left(Q_{2}\right)=Q_{2}
$$

and we have that $J\left(Q_{2}\right)<L\left(P_{2}\right)$. Again, Bolzano's Theorem concludes the proof.

Now, we analyze the stability of equilibria.

The predator-free equilibria, $Y^{*}=0$, are unstable, because the linearized equation around these points is

$$
\Pi_{3}^{\prime}(t)=\left(m+\varepsilon M_{1} I^{*}+\varepsilon M_{2} S^{*}\right) \Pi_{3}(t)
$$

where $\Pi_{3}(t):=Y(t)-Y^{*}$.

To analyze the local stability we proceed by standard linearization techniques. Linearizing (21) and after some computations the characteristic equation is

$$
\begin{aligned}
F(\lambda):= & \lambda^{3}+\left(a_{1}+b_{2}+c_{3}\right) \lambda^{2}+\left(a_{1} b_{2}+a_{1} c_{3}+b_{2} c_{3}-a_{2} b_{1}-a_{3} c_{1}-b_{3} c_{2}\right) \lambda \\
& +\left(a_{1} b_{2} c_{3}-a_{2} b_{1} c_{3}+a_{2} b_{3} c_{1}+a_{3} b_{1} c_{2}-a_{3} b_{2} c_{1}-a_{1} b_{3} c_{2}\right):=\lambda^{3}+d_{1} \lambda^{2}+d_{2} \lambda+d_{3}=0 .
\end{aligned}
$$

where

$$
\begin{aligned}
& a_{1}:=\left(-q \beta\left(P^{*}\right)+\mu_{1}\left(P^{*}\right)-q \beta^{\prime}\left(P^{*}\right) I^{*}+\mu_{1}^{\prime}\left(P^{*}\right) I^{*}-K S^{*}+M_{1} Y^{*}\right) \\
& a_{2}:=\left(-q \beta^{\prime}\left(P^{*}\right)+\mu_{1}^{\prime}\left(P^{*}\right)-K\right) I^{*} \\
& a_{3}:=M_{1} I^{*}, b_{1}:=-\beta^{\prime}\left(P^{*}\right)\left(S^{*}+(1-q) I^{*}\right)-(1-q) \beta\left(P^{*}\right)+\mu_{2}^{\prime}\left(P^{*}\right) S^{*}+K S^{*} \\
& b_{2}:=-\beta\left(P^{*}\right)-\beta^{\prime}\left(P^{*}\right)\left(S^{*}+(1-q) I^{*}\right)+\mu_{2}\left(P^{*}\right)+\mu_{2}^{\prime}\left(P^{*}\right) S^{*}+K I^{*}+M_{2} Y^{*} \\
& b_{3}:=M_{2} S^{*}, c_{1}:=-\varepsilon M_{1} Y^{*}, c_{2}:=-\varepsilon M_{2} Y^{*}, c_{3}:=m+\varepsilon M_{1} I^{*}+\varepsilon M_{2} S^{*} .
\end{aligned}
$$

We are ready to show the stability results.

Theorem 3.2. $\quad$ 1. The trivial equilibrium $(0,0, m / n)$ is $G A S$ when $F_{2}(0)>0$ and unstable when $F_{2}(0)<0$.

2. The disease-free equilibrium $\left(0, P_{S Y},\left(m+\varepsilon M_{2} P_{S Y}\right) / n\right)$ is $L A S$ when $K<K_{4}$, and unstable when $K>K_{4}$.

3. Under the assumption $K>T_{2}$, if $-\beta^{\prime}\left(P^{*}\right)\left(S^{*}+(1-q) I^{*}\right)+\mu_{2}^{\prime}\left(P^{*}\right) S^{*}>(1-q) \beta\left(P^{*}\right)$ then any endemic equilibrium $\left(I^{*}, S^{*}, Y^{*}\right)$ is LAS. 
Proof. It is not hard to see the local stability or instability of the trivial equilibrium and the disease-free equilibrium.

Now let us turn to the endemic equilibria. By the Routh-Hurwitz criterion we see that the characteristic equation does not posses any root with real positive part if, and only if,

$$
d_{i}>0 \text { for } i=1,2,3, \quad d_{1} d_{2}-d_{3}>0 .
$$

It is immediate to see that $a_{1}, a_{3}, b_{3}, c_{3}>0$, and $a_{2}, c_{1}, c_{2}<0$. Since $\left(I^{*}, S^{*}, Y^{*}\right)$ verifies $(21)_{2}$, we have

$$
b_{2}=(1-q) \beta\left(P^{*}\right) \frac{I^{*}}{S^{*}}-\beta^{\prime}\left(P^{*}\right)\left(S^{*}+(1-q) I^{*}\right)+\mu_{2}^{\prime}\left(P^{*}\right) S^{*}>0,
$$

and, by $(21)_{1}$, we get

$$
b_{1}=-\beta^{\prime}\left(P^{*}\right)\left(S^{*}+(1-q) I^{*}\right)-\beta\left(P^{*}\right)+\mu_{1}\left(P^{*}\right)+M_{1} Y^{*}+\mu_{2}^{\prime}\left(P^{*}\right) S^{*} .
$$

Moreover, from (26), it follows that

$$
\left(\mu_{1}\left(P^{*}\right)-\beta\left(P^{*}\right)+M_{1} Y^{*}\right)=\left(\mu_{1}\left(P^{*}\right)-\mu_{2}\left(P^{*}\right)+\left(M_{1}-M_{2}\right) Y^{*}\right) \frac{S^{*}}{P^{*}},
$$

and hence that $b_{1}>0$. Thus

$$
d_{1}=a_{1}+b_{2}+c_{3}>0, d_{2}=a_{1} b_{2}+a_{1} c_{3}+b_{2} c_{3}-a_{2} b_{1}-a_{3} c_{1}-b_{3} c_{2}>0 .
$$

Next we examine the sign of $d_{3}$. We have

$$
a_{3} c_{2}-a_{2} c_{3}>\left(-\varepsilon M_{1} M_{2} Y^{*}+\varepsilon M_{1} M_{2} Y^{*}\right) I^{*}=0,
$$

and so $d_{3}>0$.

To finish we need to show $d_{1} d_{2}-d_{3}>0$. Since $a_{1}>0, a_{2}>-K I^{*}, b_{1}>K S^{*}, b_{2}>0$, then

$$
d_{1} d_{2}-d_{3}>\varepsilon n M_{1}^{2} I^{*}\left(Y^{*}\right)^{2}+\varepsilon n M_{2}^{2} S^{*}\left(Y^{*}\right)^{2}>0
$$

Our next goal is to establish the global stability of the equilibrium $(0,0, m / n)$ when $F_{2}(0)>0$. Since $F_{2}(0)>0$, there exists $\delta>0$ such that

$$
\beta(P(t))-\mu_{2}(P(t))-M_{2}(m / n-\delta)<0
$$

for $t \geq 0$. By (2) we have

$$
Y^{\prime}(t) \geq(m-n Y(t)) Y(t)
$$


Thus there is $t_{0}>0$ such that $Y(t) \geq m / n-\delta$ for $t \geq t_{0}$. So, for $t \geq t_{0}$

$$
P^{\prime}(t) \leq\left(\beta(P(t))-\mu_{2}(P(t))-M_{2}(m / n-\delta)\right) P(t) .
$$

By (27), we conclude that $P(t) \rightarrow 0$ as $t \rightarrow \infty$. Moreover, we have

$$
0 \leq S(t) \leq P(t)
$$

hence $S(t) \rightarrow 0$ as $t \rightarrow \infty$. Consequently, $I(t) \rightarrow 0$ as $t \rightarrow \infty$. We may now apply the Corollary 2.6 , since $Y$ verifies the ordinary equation

$$
Y^{\prime}=\left(m-n Y+\varepsilon M_{1} I(t)+\varepsilon M_{2} S(t)\right) Y,
$$

which is asymptotically stable to $Y^{\prime}=(m-n Y) Y$, it follows that $Y(t) \rightarrow m / n$ as $t \rightarrow \infty$. This completes the proof.

Theorem 3.3. We assume $F_{2}(0)<0$, if

$$
M_{1} m>M_{1}(K):=n\left(K P_{S}-\mu_{1}(0)+q \beta(0)\right) .
$$

then the disease disappears.

In fact, the disease-free equilibrium $\left(0, P_{S Y},\left(m+\varepsilon M_{2} P_{S Y}\right) / n\right)$ is $G A S$.

Proof. By (29), there exists $\delta>0$ such that

$$
q \beta(0)-\mu_{1}(0)+K\left(P_{S}+\delta\right)-M_{1}(m / n-\delta)<0 .
$$

From (2) and (28), fixed $\delta>0$, there is $t_{0}>0$ such that for $t \geq t_{0}$

$$
\begin{aligned}
& 0 \leq P(t) \leq P_{S}+\delta \\
& Y(t) \geq m / n-\delta .
\end{aligned}
$$

In addition, by (2), there exists $t_{2}>0$ such that for $t \geq t_{2}$

$$
I^{\prime} \leq\left(q \beta(0)-\mu_{1}(0)+K\left(P_{S}+\delta\right)-M_{1}\left(\frac{m}{n}-\delta\right)\right) I .
$$

Hence, by (30), $I(t) \rightarrow 0$ as $t \rightarrow+\infty$.

Thus, the system (2) is asymptotically autonomous with

$$
\left\{\begin{array}{l}
S^{\prime}=\left(\beta(S)-\mu_{2}(S)-M_{2} Y\right) S \\
Y^{\prime}=\left(m-n Y+\varepsilon M_{2} S\right) Y .
\end{array}\right.
$$


Now, we study (31). Using the Dulac multiplier $1 / Y S$, we obtain

$$
\frac{\partial}{\partial S}\left(\frac{\beta(S)-\mu_{2}(S)}{Y}-M_{2}\right)+\frac{\partial}{\partial Y}\left(\frac{m-n Y}{S}+\varepsilon M_{2}\right)=\frac{\beta^{\prime}(S)-\mu_{2}^{\prime}(S)}{Y}-\frac{n}{S}<0,
$$

so that there are no periodic solution or cycle by Dulac's Criterion [13]. Furthermore the $\omega$ limit set of this system is $\left\{\left(P_{S Y},\left(m+\varepsilon M_{2} P_{S Y}\right) / n\right)\right\}$ by Poincaré-Bendixson [13] and the fact that in this case the other equilibria $\left(\left(P_{S}, 0\right),(0, m / n)\right.$ and $\left.(0,0)\right)$ are unstable. By Corollary 2.6 we obtain the result, i.e. the disease-free equilibrium $\left(0, P_{S Y},\left(m+\varepsilon M_{2} P_{S Y}\right) / n\right)$ is GAS.

\section{Conclusions and a specific example}

We can give the following biological interpretation to the above results.

First we consider the case without predator:

- If the net birth rate $(\beta(0))$ is less than the net death rate of the susceptible individuals $\left(\mu_{2}(0)\right)$, then the prey goes to extinction.

- On the other hand, if $\beta(0)>\mu_{2}(0)$ and

- if the rate of infection, $K$, is low enough, then infectives go to extinction, i.e the disease disappears.

- However, if $K$ is large enough then the infection persists in time.

Now, we introduce in the process a predator, i.e we consider the system (2).

- If the predation rate of susceptible individuals, $M_{2}$, or the carrying capacity of the environment, $D:=m / n$, is sufficiently large $\left(M_{2} D>\beta(0)-\mu_{2}(0)\right)$, then the predator drives the prey to extinction.

- Assume that $M_{2} D<\beta(0)-\mu_{2}(0)$, then

- if the predation rate of infective individuals, $M_{1}$, is large enough, then the disease disappears.

- However, under some assumptions (see Th. 3.2), the disease remains endemic.

As consequence, we conclude if the rate of infection, $K$, is large enough and there is not predator, the infective individuals persist; but if we include a predator with large rate of infection, the predator kills the disease but not the susceptible individuals. 
We would point out that if $F_{2}(0)<0$ then $R_{0}<0$. Moreover, in this case, we have that $P_{S Y}<$ $P_{S}$. Consequently, if there exists the disease free equilibrium in the model with predators there exists the corresponding equilibrium without predators. And, the prey population is smaller in the former case as intuitively one expects.

Now, we consider a particular case, in which we see that in the case without predator the disease persists whereas in the case with predator the disease disappears. Let

$$
\begin{aligned}
& \mu_{i}(P):=\mu_{i} * P \text { with } \mu_{1}>\mu_{2}>0, \\
& \beta(P):=e^{-\beta * P} \text { with } \beta>0 .
\end{aligned}
$$

We first examine the case without predator. In this case, we have $R_{0}=R_{1}=-1<0$. We write, $\bar{K}:=\mu_{1}-q \mu_{2}$. We obtain that $(0,0)$ is unstable. Moreover, there is another equilibrium $(0, \bar{S})$ where $\mu_{2} * \bar{S}=e^{-\beta * \bar{S}}$. The disease-free equilibrium is stable if $K<\bar{K}$ and unstable if $K>\bar{K}$. On the other hand, in this case we can improve the estimates obtained in Section 3 and we conclude that if $K>\bar{K}$, then there exists a unique endemic equilibrium $\left(I^{*}, S^{*}\right)$ which is stable.

For the case with predator, we check that there exists a unique disease-free equilibrium, $\left(0, \hat{S}, \frac{\varepsilon M_{2} \hat{S}}{n}\right)$, where $\mu_{2} * \hat{S}-e^{-\beta * \hat{S}}+M_{2} \frac{m+\varepsilon M_{2} \hat{S}}{n}=0$ if, and only if

$$
\frac{M_{2} m}{n}<1
$$

This disease-free equilibrium is stable if

$$
K<\bar{K}+\left(\frac{m+\varepsilon M_{2} \hat{S}}{n \hat{S}}\right)\left(M_{1}-q M_{2}\right)
$$

in fact by Theorem 3.3 if

$$
M_{1} m>n(K \bar{S}+q) \text { and } \frac{M_{2} m}{n}<1
$$

then it is GAS.

Summarizing, if we assume $M_{1} m>n(K \bar{S}+q)$ and $M_{2} m / n<1$, for $K>\bar{K}$ the disease persists for the case without predator and disappears for the influence of the predator.

\section{Acknowledgements}

We wish to express our sorrow at the unfortunate disappearance of the Prof. O. Arino and our gratitude by his valuable comments. 
We are delighted to thank to the referee for his/her careful reading of the manuscript improving the presentation of the paper.

\section{References}

1. Lotka AJ. Elements of Mathematical Biology. Dover, 1956.

2. Von Foerster H. Some remarks on changing populations. The Kinetics of Cellular Proliferation, 1959: 382-407.

3. Gurtin ME, MacCamy RC. Non-linear age-dependent population dynamics. Arch. Ration. Mech. Anal. 1974; 54: 281-300, .

4. Webb GF. Theory of nonlinear Age-dependent Population Dynamics. Pure Appl. Math. Monographs, Marcel Dekker, New York, 1985.

5. Hethcote HW. The mathematics of infectious diseases. SIAM Rev. 2000; 42: 599-653.

6. Chattopadhyay J, Arino O. A predator-prey model with disease in the prey. Nonlinear Anal. 1999; 36: 747-766.

7. Han L, Ma Z, Hethcote HW. Four predator prey models with infectious diseases. Math. Comput. Modelling 2001; 34: 849-858.

8. Venturino E. Age-structured predator-prey models. Math. Modelling 1984; 5: 117-128.

9. Venturino E. The effects of diseases on competing species. Math. Biosci. 2001; 174: $111-131$.

10. Chattopadhyay J, Pal S, El Abdllaoui A. Classical predator-prey system with infection of prey population-a mathematical model. Math. Methods Appl. Sci. 2003; 26: 1211-1222.

11. Arino O, Delgado M, Molina-Becerra M. Asymptotic behavior of disease-free equilibrium solutions of an age-structured predator-prey model with disease in the prey. Discrete Contin. Dyn. Syst. Ser. B 2004; 4: 501-515.

12. Thieme HR. Convergence results and a Poincaré-Bendixson trichotomy for asymptotically autonomous differential equations. J. Math. Biol. 1992; 30:755-763.

13. Perko L. Differential Equations and Dynamical Systems, volume 7 of Texts in Applied Mathematics. Springer-Verlag, New York, third edition, 2001. 